\title{
OPEN-CIRCUIT END EFFECT OF MICROSTRIP LINE CONFIGURATION IN PLASMA MEDIUM
}

\author{
PREM BHUSHAN MITAL, \\ Fellow, IETE \\ Department of Electronics \& Communication Engineering, $C R$ State College of Engineering, Murthal \\ (Sonepat) Pin 131039 (INDIA)
}

(Received August 31, 1995; in final form January 25, 1996)

The extension in length for open microstrip configuration in plasma media is determined using spectral domain technique under quasi static approach $[1,2]$. The results were verified by modifying Hammerstad equation for plasma media. Good agreement is found between the two results.

\section{INTRODUCTION}

The ideal field patterns of a open-circuited microstrip line are distorted with fringing electric fields. The fringing fields and the increase in electrostatic energy as a result of the extra stored energy is modeled by a capacitive termination $\mathrm{C}_{\mathrm{F}}$. The fringing capacitance at the termination of the line is equivalent to extending line by $\Delta \ell$ as given by the expression

$\Delta \ell=\frac{1}{\beta} \tan ^{-1}\left(\mathrm{Z}_{0} \omega \mathrm{C}_{\mathrm{F}}\right)$

where

$\beta=$ propagation constant on the line

$Z_{0}=$ characteristic impedence of the line

$\omega=$ angular frequency

\section{FORMULATION}

The microstrip in plasma medium is shown in Fig. 1 where $\epsilon_{\mathrm{p}}$ is the dielectric constant of plasma medium defined as

$\epsilon_{p}=\mathrm{A}^{2}=1-\left(\frac{\omega_{p}}{\omega}\right)^{2}$

where $\mathrm{A}$ is the plasma parameter

If $\phi(x, y)=$ static potential distribution in microstrip structure 


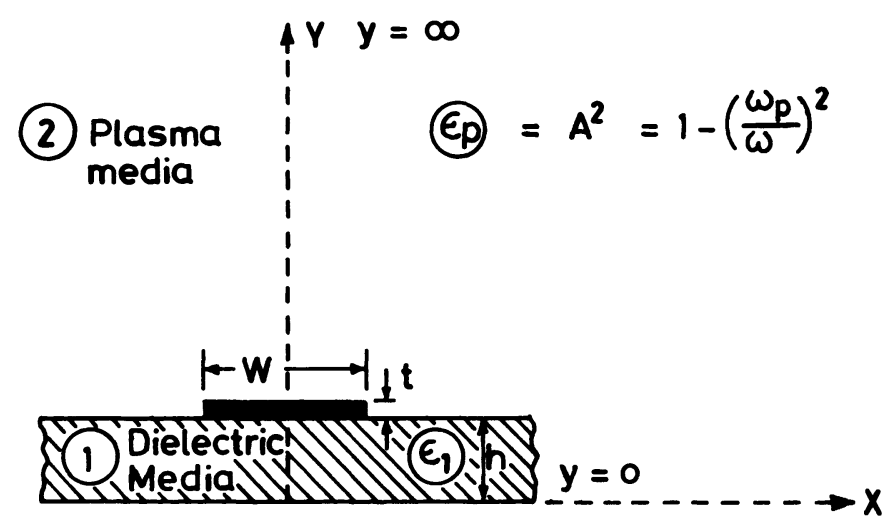

\section{MICROSTRIP IN PLASMA MEDIUM \\ FIGURE 1}

then $\tilde{\phi}(\beta, y)=$ Fourier transform of $\phi(x, y)$

$$
=\int_{-\infty}^{\infty} \phi(x, y) e^{j \beta x} d x
$$

Assuming in media (1) $\phi=\mathrm{Ae}^{\beta y}+\mathrm{Be}^{-\beta y}$

$\&$ in media (2) $\quad \phi=\mathrm{Ce}^{-\beta y}+\mathrm{De}^{\beta y}$

In Fourier transform domain, the boundary conditions are

$$
\begin{aligned}
\text { at } y=0 & \tilde{\phi}(\beta, 0)=0 \\
y=\infty & \tilde{\phi}(\beta, \infty)=0
\end{aligned}
$$

At interface

$\tilde{\phi}(\beta, h+0)=\tilde{\phi}(\beta, h-0)$

and $\epsilon_{\mathrm{p}} \frac{\mathrm{d} \tilde{\phi}}{\mathrm{dy}}(\beta, \mathrm{h}+0)=\epsilon_{1} \frac{\mathrm{d}}{\mathrm{dy}} \tilde{\phi}(\beta, \mathrm{h}-0)-\tilde{\mathrm{f}}(\beta)$

Taking $f(x)$ as the change distribution on the strip conductor, total charge on strip conductor

$Q=\int_{-\infty}^{\infty} f(x) d x$

Hence 
$\tilde{f}(\beta)=\int_{-\infty}^{\infty} f(x) e^{j \beta x} d x$

Solving the above, we have

$\Phi(\mathbf{x}, \mathrm{h})=\mathrm{Ce}^{-\beta \mathrm{h}}=\frac{\tilde{\mathrm{f}}(\beta)}{\beta\left(\epsilon_{1} \operatorname{coth} \beta \mathrm{h}+\epsilon \mathrm{p}\right)}$

Hence the fourier transform of potential distribution is given as:

$\tilde{\phi}(\beta, h)=\frac{\tilde{f}(\beta)}{\beta\left(\epsilon_{1} \operatorname{coth} \beta h+e p\right)}$

Line capacitance $\mathrm{C}$ is obtained using symmetry as

$\frac{1}{C}=\frac{1}{\pi \epsilon Q^{2}} \int_{0}^{\infty} \frac{\{\tilde{f}(\beta)\}^{2}}{\left\{\epsilon p+\epsilon_{1} \operatorname{coth} \beta h\right\} \beta h} d(\beta h)$

Taking approximately trial function for $f(x)=|x|$ which gives capacitance as maximum

$f(x)=\left\{\begin{array}{l}|x|-\omega / 2 \leq x \leq \omega / 2 \\ 0 \text { elsewhere }\end{array}\right.$

$\frac{\tilde{f}(\beta)}{Q}=2\left[\frac{2 \sin \left(\frac{\beta \omega}{2}\right)}{\left(\frac{\beta \omega}{2}\right)}\right]-\left[\frac{\sin \left(\frac{\beta \omega}{4}\right)}{\frac{\beta \omega}{4}}\right]^{2}$

If $\beta h=x$, then $d \beta h=d x$.

Hence

$\frac{1}{C}=\frac{\frac{1}{\pi \epsilon_{0}} \int\left[\frac{f\left(\frac{x}{h}\right)}{Q}\right]^{2}}{\left[\epsilon p+\epsilon r_{1} \operatorname{coth} x\right] x} d x$

or 


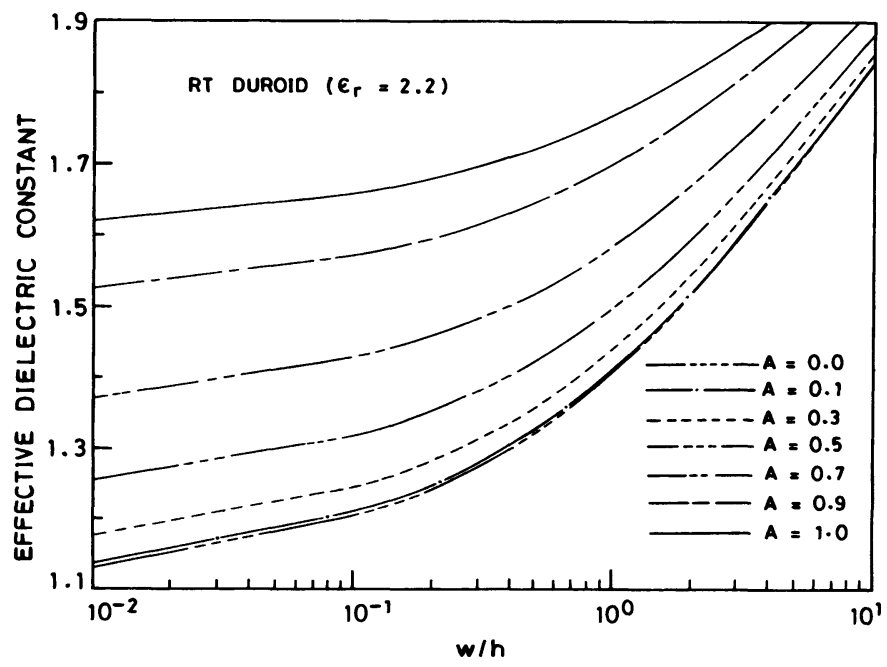

FIGURE 2

$$
\frac{\mathrm{C}}{\epsilon_{0}}=\frac{\pi\left[\frac{\sin \left(\frac{\mathrm{x}}{2} \frac{\omega}{\mathrm{h}}\right)}{\frac{\mathrm{x}}{2} \frac{\omega}{\mathrm{h}}}\right]-\left[\frac{\sin \frac{\mathrm{x}}{4} \frac{\omega}{\mathrm{h}}}{\frac{\mathrm{x}}{4} \frac{\omega}{\mathrm{h}}}\right]^{2}}{\left(\epsilon \mathrm{p}+\epsilon_{1} \operatorname{coth} \mathrm{x}\right) \mathrm{x}}
$$

The effective permittivity of microstrip is given by

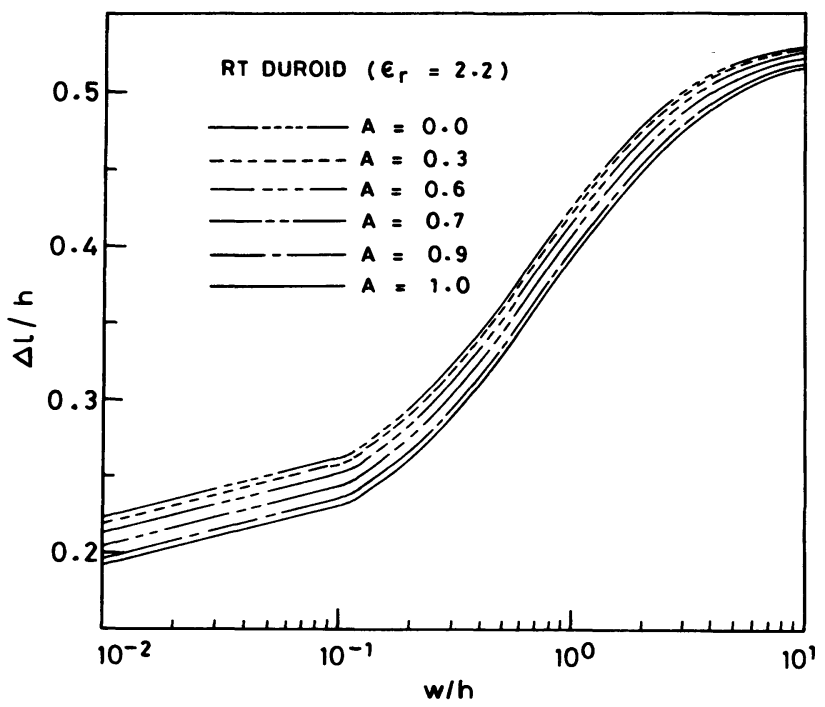

FIGURE 3 
$\epsilon_{\text {eff }}=\frac{\mathrm{C}}{\mathrm{C}_{0}}$

where $\mathrm{C}$ and $\mathrm{C}_{0}$ are the capacitances per unit length of line with and without dielectric. The effective dielectric constant is also given as

$\epsilon_{\mathrm{eff}}=\frac{\epsilon_{\mathrm{r}}+\epsilon \mathrm{p}}{2}+\frac{\epsilon_{\mathrm{r}}-\epsilon \mathrm{p}}{2} \frac{1}{\sqrt{1+\frac{12 \mathrm{~h}}{\omega}}} \frac{\omega}{\mathrm{h}} \geq 1$

And line extension $\Delta \ell$ as given by Hammerstad is [3]:

$$
\frac{\Delta \ell}{\mathrm{h}}=0.412 \cdot \frac{\epsilon_{\mathrm{e}}+0.3}{\epsilon_{\mathrm{e}}-0.258} \cdot \frac{\frac{\omega}{\mathrm{h}}+0.262}{\frac{\omega}{\mathrm{h}}+0.813}
$$

\section{NUMERICAL RESULTS}

The results were obtained solving equations $15,16,17$, and 18 numerically and are plotted in Fig. 2 to 5.

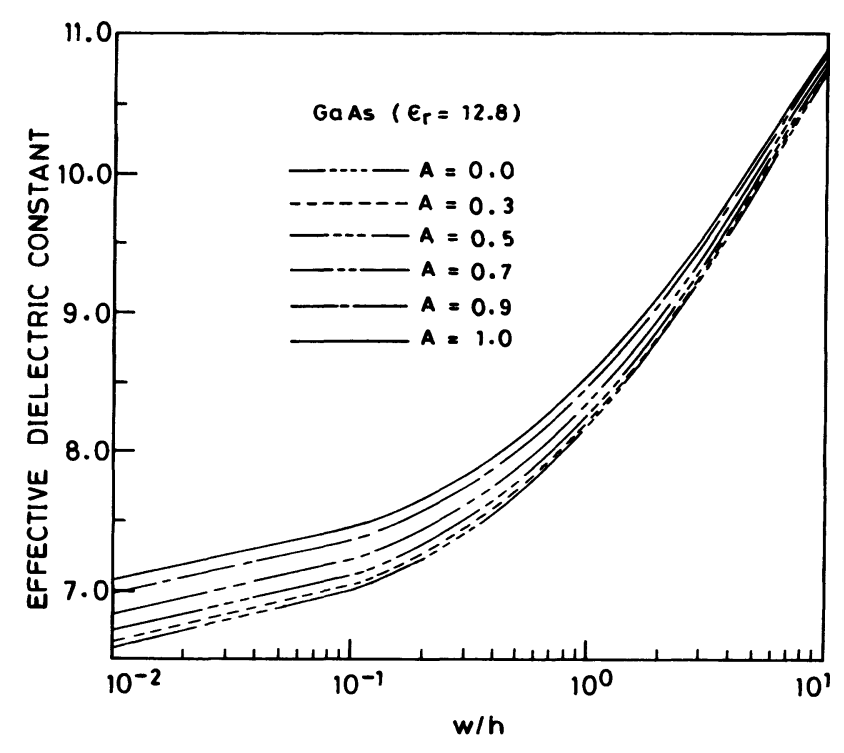

FIGURE 4 


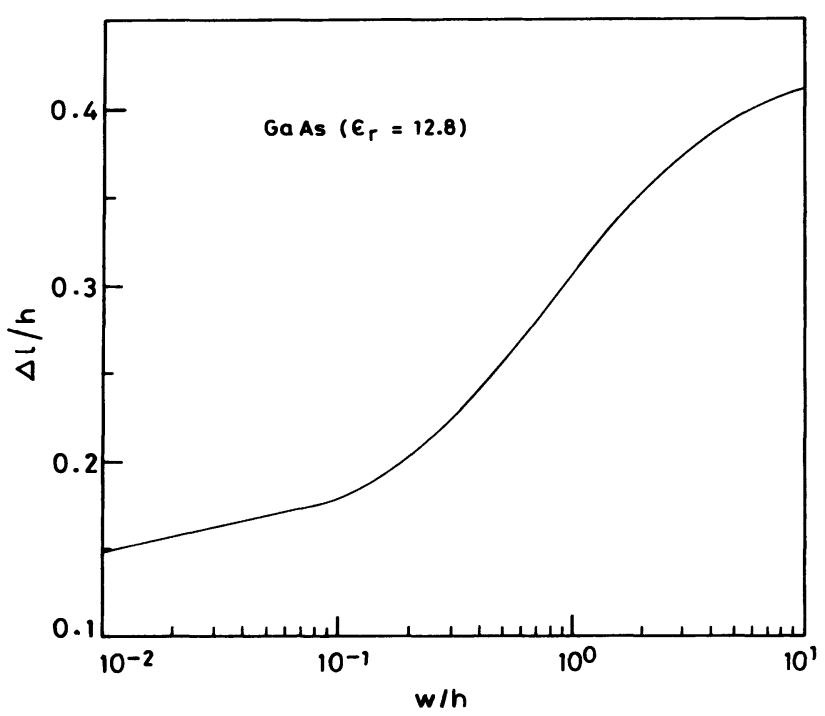

FIGURE 5

\section{CONCLUSIONS}

Calculations of length extension $(\Delta \ell)$ in plasma medium for various plasma parameter values $(\mathrm{A}=.1,3, .5,7, .9)$ in an open microstrip configuration are obtained using spectral domain technique under quasi static approach $[4,5]$ and by modifying Hammerstad equation for plasma media. Good agreement is found between the two results.

\section{ACKNOWLEDGEMENT}

The author is indebted to Prof. B. Bhat and Prof. S.K. Koul, Centre for Applied Research in Electronics, I.I.T. New Delhi for providing research facilities.

\section{REFERENCES}

1. E. Yamashita "Variational method for the analysis of Microstrip-like transmission lines", IEEE trans. Microwave theory and techniques, Vol. MTT-16, pp. 529-535, August 1968.

2. B. Bhat \& S.K. Koul "Lumped Capacitance, Open circuit end effects and edge capacitance of microstrip-like transmission lines for microwave and millimeter wave applications", IEEE trans., Microwave theory and techniques, Vol. MTT-32, No. 4, pp. 433-439, April 1984.

3. E.O. Hammerstad "Equations for Microstrip Circuit design", 5th European MW conference, pp. 268-272, 1975 .

4. P. Silvester and P. Benedek, "Equivalent capacitance of Microstrip Open Circuits", IEEE trans. MTT-20, pp. 511-516.

5. B. Bhat \& S.K. Koul, "Strip line-like transmission lines for Microwave Integrated Circuits", Willey Eastern Ltd. 

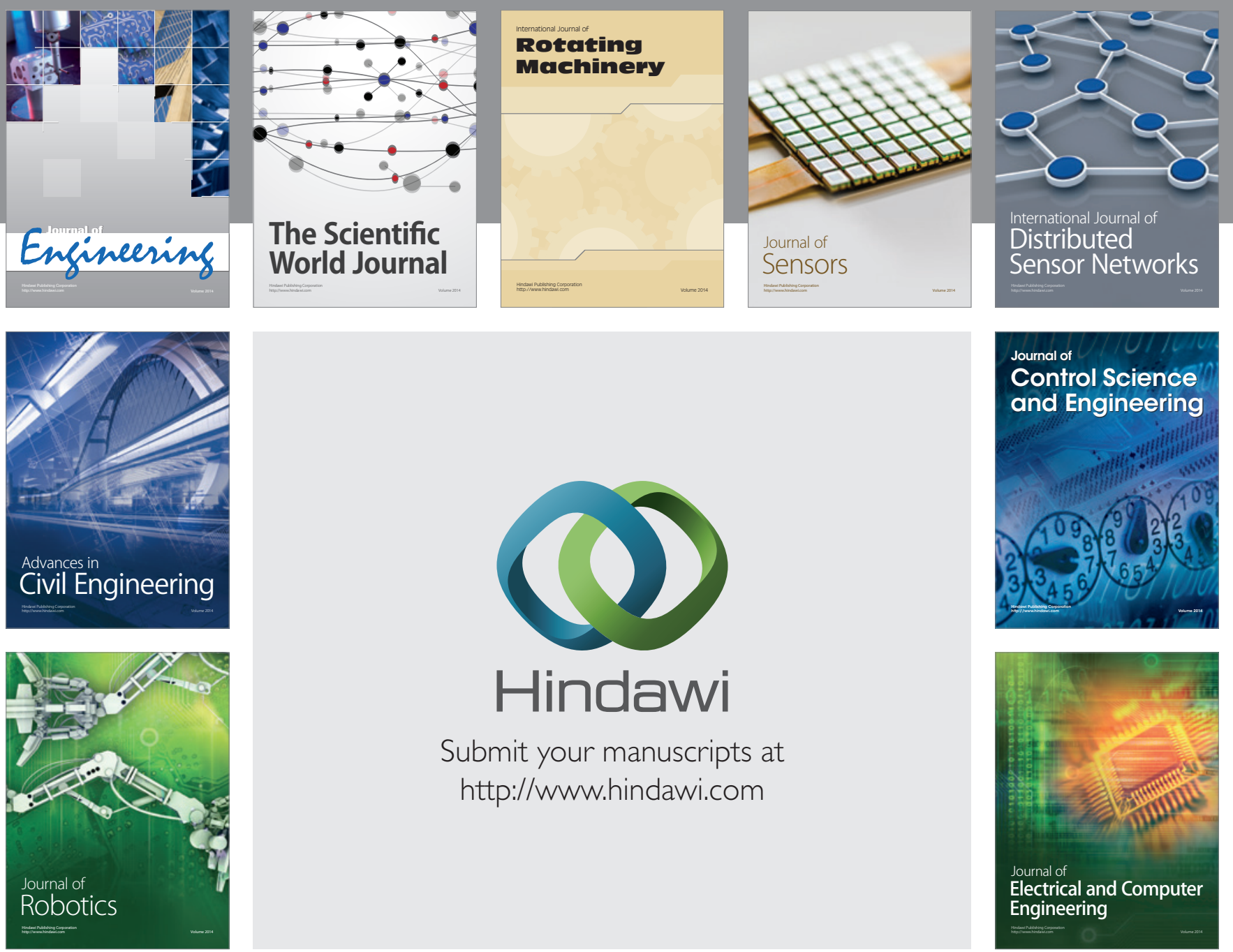

Submit your manuscripts at

http://www.hindawi.com
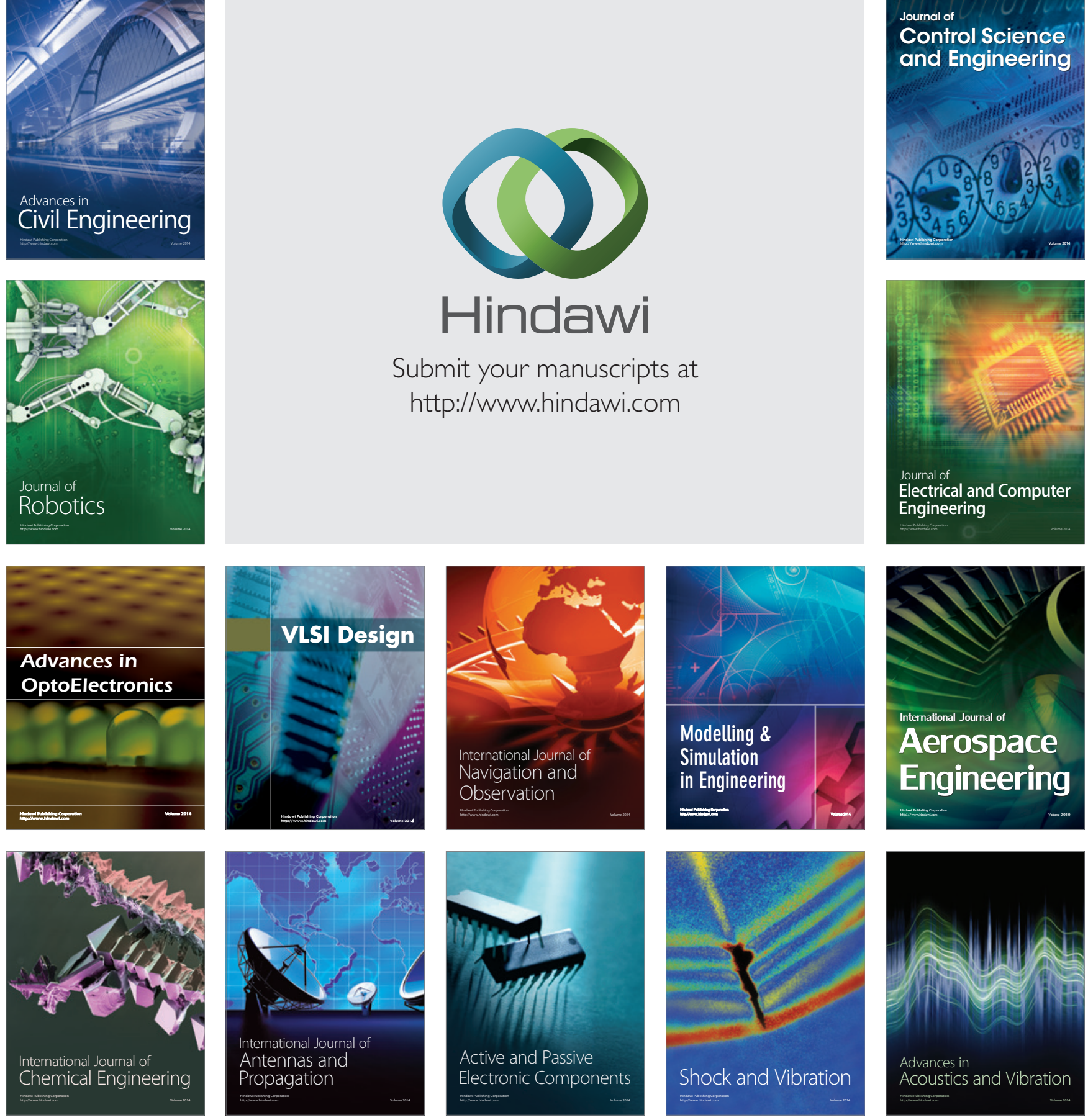